\title{
Out-of-Equilibrium Kondo Effect in a Mesoscopic Device
}

\author{
S. De Franceschi, ${ }^{1}$ R. Hanson, ${ }^{1}$ W. G. van der Wiel,,${ }^{1,2}$ J. M. Elzerman,${ }^{1}$ J. J. Wijpkema, ${ }^{1}$ T. Fujisawa, ${ }^{3}$ \\ S. Tarucha, ${ }^{2,3}$ and L. P. Kouwenhoven ${ }^{1}$ \\ ${ }^{1}$ Department of NanoScience, DIMES, and ERATO Mesoscopic Correlation Project, Delft University of Technology, \\ P.O. Box 5046, 2600 GA Delft, The Netherlands \\ ${ }^{2}$ ERATO Mesoscopic Correlation Project, University of Tokyo, Bunkyo-ku, Tokyo 113-0033, Japan \\ ${ }^{3}$ NTT Basic Research Laboratories, Atsugi-shi, Kanagawa 243-0198, Japan
}

(Received 13 February 2002; published 20 September 2002)

\begin{abstract}
We study the nonequilibrium regime of the Kondo effect in a quantum dot laterally coupled to a narrow wire. We observe a split Kondo resonance when a finite bias voltage is imposed across the wire. The splitting is attributed to the creation of a double-step Fermi distribution function in the wire. Kondo correlations are strongly suppressed when the voltage across the wire exceeds the Kondo temperature. A perpendicular magnetic field enables us to selectively control the coupling between the dot and the two Fermi seas in the wire. Already at fields of order $0.1 \mathrm{~T}$ only the Kondo resonance associated with the strongly coupled reservoir survives.
\end{abstract}

DOI: 10.1103/PhysRevLett.89.156801

PACS numbers: 73.23.-b, 73.63.Kv

Mesoscopic devices form a powerful tool for the study of fundamental many-body phenomena. A striking example is the Kondo effect in a quantum-dot device, which consists of a small electronic island connected by tunable tunnel barriers to extended leads [1]. Predicted in 1988 [2], the Kondo effect in a quantum dot was observed for the first time ten years later [3], leading to intense research [4]. Quantum dots offer new control to study various aspects of the Kondo effect. One option is the possibility to explore nonequilibrium regimes, e.g., by applying a finite voltage between the leads connected to the dot.

At equilibrium, Kondo correlations give rise to a sharp resonance in the density of states (DOS). The resonance is aligned with the Fermi energy of the leads. For a finite bias voltage the resonance is predicted [5] to split as shown qualitatively in Fig. 1(a). The resulting two peaks are aligned with the Fermi energies of the leads and become progressively smaller as the bias voltage is increased. This suppression is due to decoherence introduced by the inelastic scattering of electrons from the high- to the low-energy lead [5-7].

Measuring differential conductance, $d I / d V$, as a function of bias voltage, $V$, results in a nonlinear characteristic with a single peak at zero bias [5]. This zero-bias anomaly, observed in a variety of experiments, is a characteristic signature of the Kondo effect. However, a $d I / d V$-vs- $V$ measurement does not provide enough information to extract the $V$-dependent DOS. In particular, whether the Kondo resonance is indeed split and how the resulting double-peak DOS evolves with bias is an issue that has not yet been experimentally investigated. Whether Kondo correlations survive at voltages larger than the Kondo temperature at equilibrium is an issue that has recently raised considerable debate [6-10]. Some authors [8,9] have argued the possibility of a two-channel Kondo effect that would develop at finite bias. A direct measurement of the DOS would contribute important information to understand Kondo physics out of equilibrium. Recent theoretical papers [11,12] have proposed to use three-terminal quantum dots in which one of the leads serves as a weakly coupled electrode to probe the local DOS. Here we address this issue from a different angle.

We couple a quantum dot to the middle of a quasiballistic quantum wire that is brought out of equilibrium by an applied bias voltage. The finite voltage creates inside the wire two ensembles of electrons (i.e., right and left movers) whose quasielectrochemical potentials, $\mu_{S 1}$ and
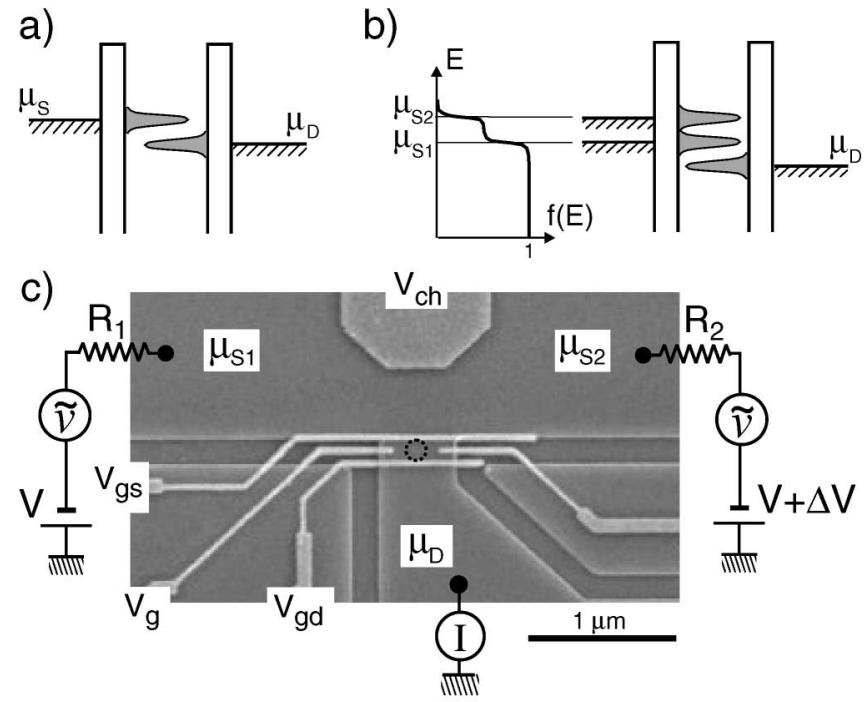

FIG. 1. (a) Splitting of the Kondo resonance due to finite bias voltage across the quantum dot. The resulting split peaks in the density of states are aligned with the Fermi energies of the leads. (b) Additional splitting due to a double-step distribution function in the source reservoir. (c) Scanning electron micrograph of the device and measurement scheme. Light grey corresponds to metallic gates, dark grey to etched regions. 
$\mu_{S 2}$, are defined by the electron reservoirs connected to the wire. Accordingly, the electron distribution function in the wire develops two steps located at $\mu_{S 1}$ and $\mu_{S 2}$ $[13,14]$ (see Fig. 1(b), left side). Our main goal is to show that the Kondo resonance splits for $\mu_{S 1} \neq \mu_{S 2}$ (see Fig. 1(b), right side).

The sample designed for this experiment was fabricated on a GaAs/AlGaAs heterostructure containing a two-dimensional electron gas (2DEG) $90 \mathrm{~nm}$ below the surface. (The experiment was reproduced on a sample with similar geometry, fabricated on a different heterostructure.) As shown in Fig. 1(c), the device has a small quantum dot (indicated by a dotted circle), formed inside a 500-nm-wide region defined by shallow etching. Confinement in the longitudinal direction is accomplished by applying negative gate voltages, $V_{g s}$ and $V_{g d}$. These gate voltages define the tunnel coupling between the quantum dot and its 2DEG leads. A third gate voltage, $V_{g}$, is used to control the electrostatic potential on the dot. On the top side, the dot is connected to the middle of a short quantum wire formed in the 2DEG by applying a voltage $V_{c h}=-0.4 \mathrm{~V}$ to a large gate coming from above. The wire then has a resistance, $R_{c h}$, of about $1.3 \mathrm{k} \Omega$, corresponding to $\sim 10$ spin-degenerate propagating modes. There are in total three regions of 2DEG connected by Ohmic contacts to the external circuitry. The bottom 2DEG (drain) is connected to the ground via a currentvoltage converter that measures the current through the quantum dot. The other two regions, source 1 and source 2, are connected to dc voltages $-V$ and $-(V+\Delta V)$.

An additional ac voltage $\tilde{\boldsymbol{v}}$ with a rms amplitude of $2.5 \mu \mathrm{V}$ is applied to both source leads allowing for lockin measurements. The resistances of the leads, $R_{1}$ and $R_{2}$, are in the $0.5-0.8 \mathrm{k} \Omega$ range, including the contribution from the respective 2DEGs. We call $\mu_{S 1}$ and $\mu_{S 2}$ the Fermi energies of the source 2DEGs, and $\mu_{D}$ the Fermi energy of the drain 2DEG.

Measurements were done in a dilution refrigerator at $15 \mathrm{mK}$. The quantum-dot parameters $\left(V_{g s}, V_{g d}\right.$, and $\left.V_{g}\right)$ were tuned to achieve a robust Kondo regime, with roughly equal tunnel rates through the top and the bottom barrier $(\sim 30 \mathrm{GHz})$. In the inset to Fig. 2(a) we show the linear conductance, $G$, of the dot as a function of $V_{g}(V$ and $\Delta V$ are set to zero). The Kondo regime (see arrow) takes place between the two Coulomb blockade peaks. Here the valley conductance is enhanced. By sweeping $V$ for $\Delta V=0$ we measure $d I / d V$ as a function of bias voltage $V$ across the dot. As in several earlier experiments, we find a narrow peak at zero bias (Fig. 2(a)) which reflects the existence of a Kondo resonance at the Fermi energy. The peak has a full width at half maximum, $w_{0} \simeq 25 \mu \mathrm{V}$, yielding a Kondo temperature $T_{K} \sim$ $e w_{0} / k_{B}=0.3 \mathrm{~K}$.

In Figs. 2(b), 2(d), and 2(f) we show on gray scale $d I / d V$ vs $\left(V, V_{g}\right)$. Each plot is obtained from many $d I / d V$ vs $V$ traces taken for a set of closely spaced $V_{g}$ values ranging between -65 and $-20 \mathrm{mV}$. Figure $2(\mathrm{~b})$, taken at
$\Delta V=0$, shows a Coulomb diamond (indicated by dotted lines) with a clearly distinguishable peak at zero bias. A nonzero $\Delta V$ results in a current $I_{12}$ between source 1 and source 2 , and a finite voltage $\left(\mu_{S 2}-\mu_{S 1}\right) / e$ across the wire. Hence the quantum dot faces, at its topside, a nonequilibrium electron distribution with two quasi-Fermi energies, $\mu_{S 1}$ and $\mu_{S 2}$, associated with the carriers from the left and right reservoirs [see Figs. 1(b) and 1(c)]. Figure 2(c) shows a $d I / d V$ vs $V$ trace taken in the middle of the Kondo valley for $\Delta V=-150 \mu \mathrm{V}$. Strikingly, the Kondo peak has split due to the applied voltage $\Delta V$. The splitting is seen all over the Coulomb diamond as shown in Fig. 2(d). A similar result is found for $\Delta V=150 \mu \mathrm{V}$ (Figs. 2(e) and 2(f)). In this case, however, the split peaks are not fully resolved [15]. As we show below, the split peaks occur at those voltages, $V$, for which $\mu_{S 1}$ or $\mu_{S 2}$ line up with $\mu_{D}$. This represents the first observation of a bias-induced splitting of the Kondo resonance. We point out that, due to the strong coupling of the drain lead,
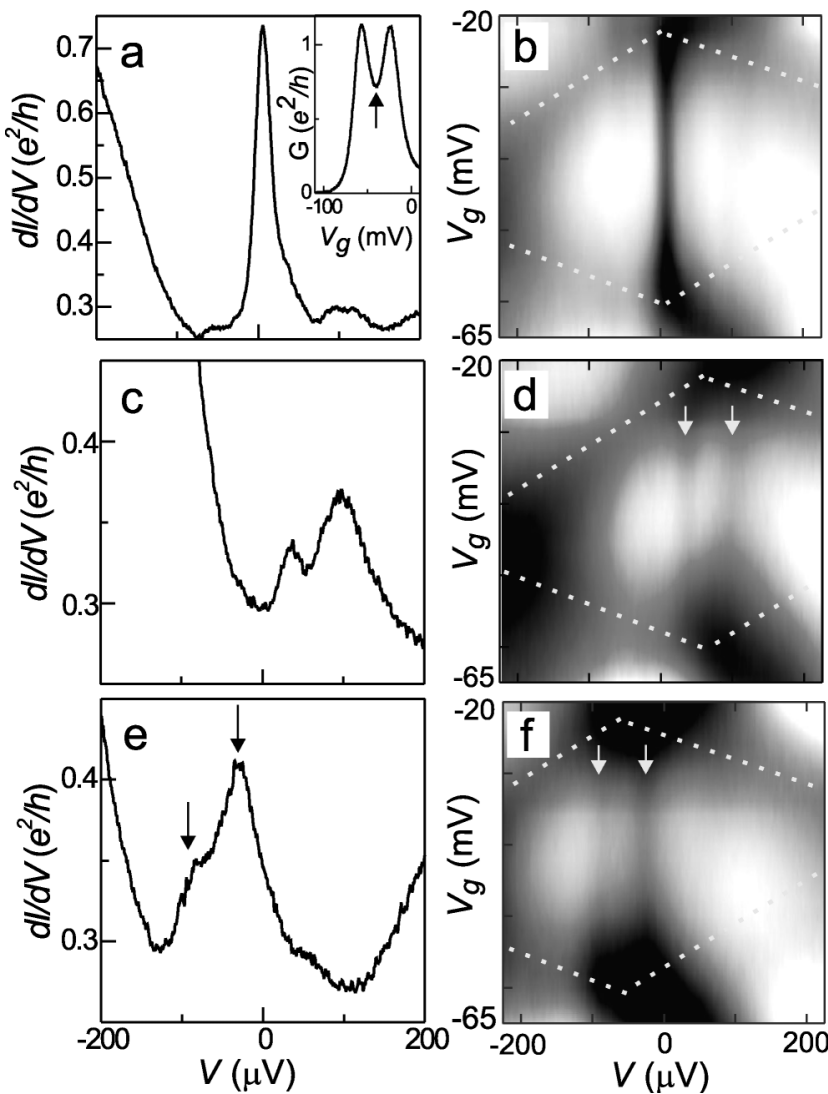

FIG. 2. (a),(c),(e) Differential conductance, $d I / d V$, vs bias voltage, $V$, across the dot. The plunger gate is at $V_{g}=-40 \mathrm{mV}$. The voltage $\Delta V$ between source 1 and source 2 is set to 0 (a), -150 (c), and $150 \mu \mathrm{V}$ (e). Inset to (a): linear conductance, $G$, vs $V_{g}$ around the Kondo valley (see arrow). (b),(d),(f) $d I / d V$ vs $\left(V, V_{g}\right)$ on gray scale. Dark gray corresponds to large $d I / d V$. $\Delta V=0$ (b), $-150 \mu \mathrm{V}$ (d), and $150 \mu \mathrm{V}$ (f). Dotted lines identify the edges of the Coulomb diamond. The Kondo anomaly shows up as a single vertical line at zero bias in (a) and as a vertical split line (see arrows) in (d) and (f). 
$d I / d V$ cannot be directly identified with the local DOS, which is in fact $V$ dependent. Yet $d I / d V$ provides the qualitative profile of the local DOS induced by the coupling to the wire electrons, similarly to the hypothetical case of a weakly coupled (drain) probe (for an in-depth discussion, see Ref. [12]).

In Fig. 3(a) we plot several $d I / d V$ vs $V$ traces taken again in the middle of the Kondo valley, but now for different $\Delta V$ between -300 and $300 \mu \mathrm{V}$. To compensate for the series resistance $R_{1}$, each trace has been shifted horizontally by as much as $R_{1} I_{12}=R_{1} \Delta V /\left(R_{1}+R_{2}+\right.$ $\left.R_{c h}\right)$, i.e., $V \rightarrow V+0.20 \Delta V$. Then $V$ becomes the voltage $\mu_{S 1} / e \simeq\left(\mu_{S 1}-\mu_{D}\right) / e$ (because of the relatively small current through the drain lead we can neglect the voltage drop on its series resistance and assume $\mu_{D}=0$ ).

The splitting of the Kondo resonance is proportional to $\Delta V$ as emphasized by the dotted lines in Fig. 3(a). One of the split peaks stays at zero bias, corresponding to the lineup condition between $\mu_{S 1}$ and $\mu_{D}$. The other peak moves with $\Delta V$ following the condition $V=\left(\mu_{S 1}-\right.$ $\left.\mu_{S 2}\right) / e=R_{c h} \Delta V /\left(R_{1}+R_{2}+R_{c h}\right)$. This corresponds to the alignment of $\mu_{S 2}$ with $\mu_{D}$.

Increasing $|\Delta V|$ [i.e. moving to the top or bottom of Fig. 3(a)] results in a suppression of the split Kondo resonance. The suppression is due to inelastic scattering processes in the wire which transfer electrons from the high- to the low-energy Fermi reservoir. On what voltage scale is the Kondo resonance washed out? To address this question we plot normalized peak height as a function of $\Delta \tilde{\mu} \equiv\left(\mu_{S 2}-\mu_{S 1}\right) / w_{0}$ [see solid circles in Fig. 3(c)]. Since $e w_{0} \sim k_{B} T_{K}$, we are scaling the voltage across the wire in units of the Kondo temperature. For $|\Delta \tilde{\mu}| \simeq 1$ (i.e., $\left|\mu_{S 2}-\mu_{S 1}\right| \sim k_{B} T_{K}$ ) the peak height becomes half of the value for $\Delta V=0$, which means that the Kondo resonance is suppressed on a voltage scale $k_{B} T_{K} / e$. For $|\Delta \tilde{\mu}|>2$ we have fitted the data to the analytical function $A \ln ^{-2}(\alpha|\Delta \tilde{\mu}|)\left[1+2 \ln ^{-1}(\alpha|\Delta \tilde{\mu}|)\right]$, derived in Ref. [7] in the limit $|\Delta \tilde{\mu}| \gg 1$. Although the latter condition is not fully satisfied we find good agreement for $A \sim 1$ and $\alpha \sim 5$.

We now discuss the effect of a magnetic field, $B$, perpendicular to the 2DEG. Already for fields of order $0.1 \mathrm{~T}$, the Lorentz force causes a significant shift in the electron waves traveling through the wire. (We note that the magnetic length for $B=0.1 \mathrm{~T}$ is about $80 \mathrm{~nm}$, i.e., a few times smaller than the effective width of the wire.) For $B<0$, left moving electrons are pushed away from the dot, whereas right movers travel closer to it [Fig. 4(a)]. The situation is reversed for $B>0$ [Fig. 4(b)].

In Figs. 4(c) and 4(d) we show $d I / d V$ vs $V$ traces taken for $B=-0.2$ and 0.2 T, respectively. As in Fig. 3(a), $V_{g}$ is adjusted to the middle of the Kondo valley, and $\Delta V$ is varied between -300 and $300 \mu \mathrm{V}$. The voltage is compensated by $0.20 \Delta V$ for the series resistance $R_{1}$. As opposed to the results for $B=0$, no splitting is observed at finite $\Delta V$. We explain such a different behavior as follows. At $B=-0.2 \mathrm{~T}$ only right movers couple effec-
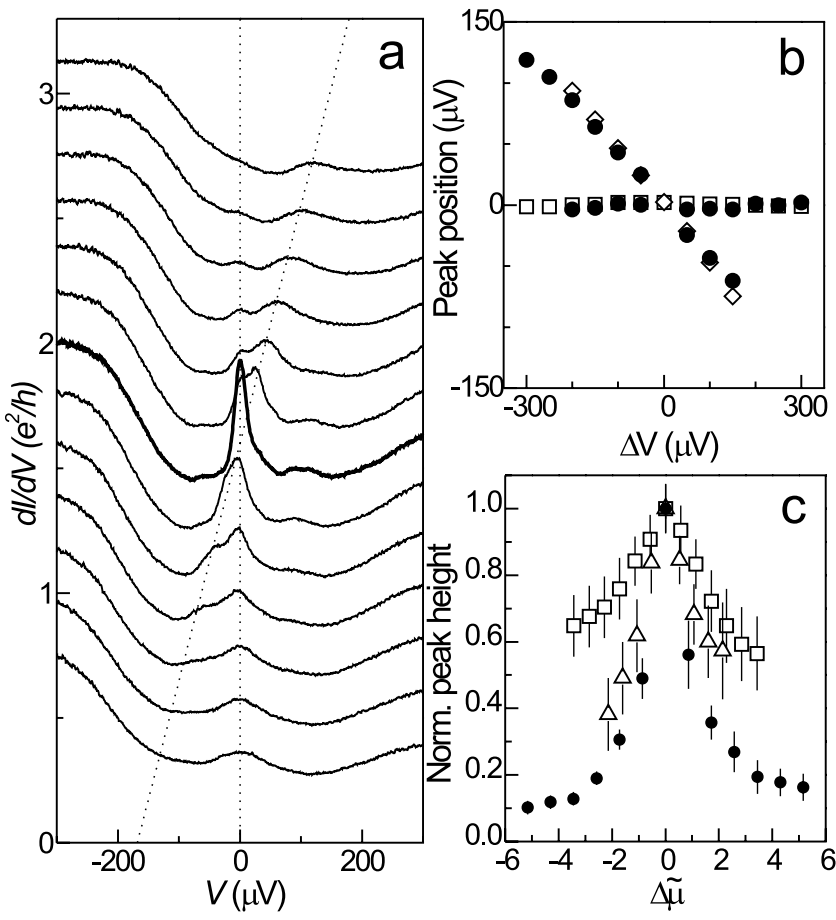

FIG. 3. (a) $d I / d V$ vs $V$, for $\Delta V$ between $-300 \mu \mathrm{V}$ (top trace) and $300 \mu \mathrm{V}$ (bottom trace) in steps of $50 \mu \mathrm{V}$. Curves are vertically offset by $0.2 e^{2} / h$. The voltage scale is shifted by $0.20 \Delta V$ to compensate for the voltage drop on $R_{1}$. The zerobias Kondo peak at $\Delta V=0$ (thick trace) splits and weakens at finite $\Delta V$. Dotted lines are drawn to emphasize the splitting. (b) $V$ position of the Kondo peaks at $B=0$ (solid circles), $B=$ $-0.2 \mathrm{~T}$ (open squares), and $B=0.2 \mathrm{~T}$ (open diamonds). (c) Height of the Kondo peaks, normalized to the $\Delta V=0$ value, vs $\Delta \tilde{\mu} \equiv\left(\mu_{S 2}-\mu_{S 1}\right) / e w_{0} . w_{0}$ is the full width at half maximum of the Kondo peak at $\Delta V=0$. For $B=0$ (solid circles), peak heights are obtained from fitting to a double Lorenztian and a linear background. For each $\Delta V$ we plot only the height of the largest peak. For $B=-0.2 \mathrm{~T}$ (open squares) and $0.2 \mathrm{~T}$ (open triangles), peak heights are obtained from fitting to a Lorentzian and a 5th order polynomial background.

tively to the dot, while the coupling for left movers is strongly suppressed [see Fig. 4(a)]. As a result, most of the current through the dot comes from the strongly coupled reservoir [see the inset to Fig. 4(c) and its relative caption] and only the Kondo peak corresponding to the lineup between $\mu_{S 1}$ and $\mu_{D}$ survives. Indeed the Kondo peak stays at $V=0$, irrespective of $\Delta V$. For $B=0.2 \mathrm{~T}$, only electrons from the right reservoir couple effectively to the dot giving rise to Kondo effect. The resulting Kondo peak occurs when $\mu_{S 2}$ is aligned with $\mu_{D}$, and its position shifts proportionally to $\Delta V$.

In the inset to Fig. 4(d) we show that the coupling of the weakly coupled lead can be restored by making the voltage $V_{c h}$ more negative. This reduces the effective width of the wire pushing together left and right movers. The splitting of the Kondo resonance by a finite $\Delta V$ is recovered for $B=0.2 \mathrm{~T}$, similarly to the case $B=0$.

Finally, we compare quantitatively results for $B=$ $\pm 0.2 \mathrm{~T}$ and $B=0$. Figure 3 (b) shows the $V$ positions of 


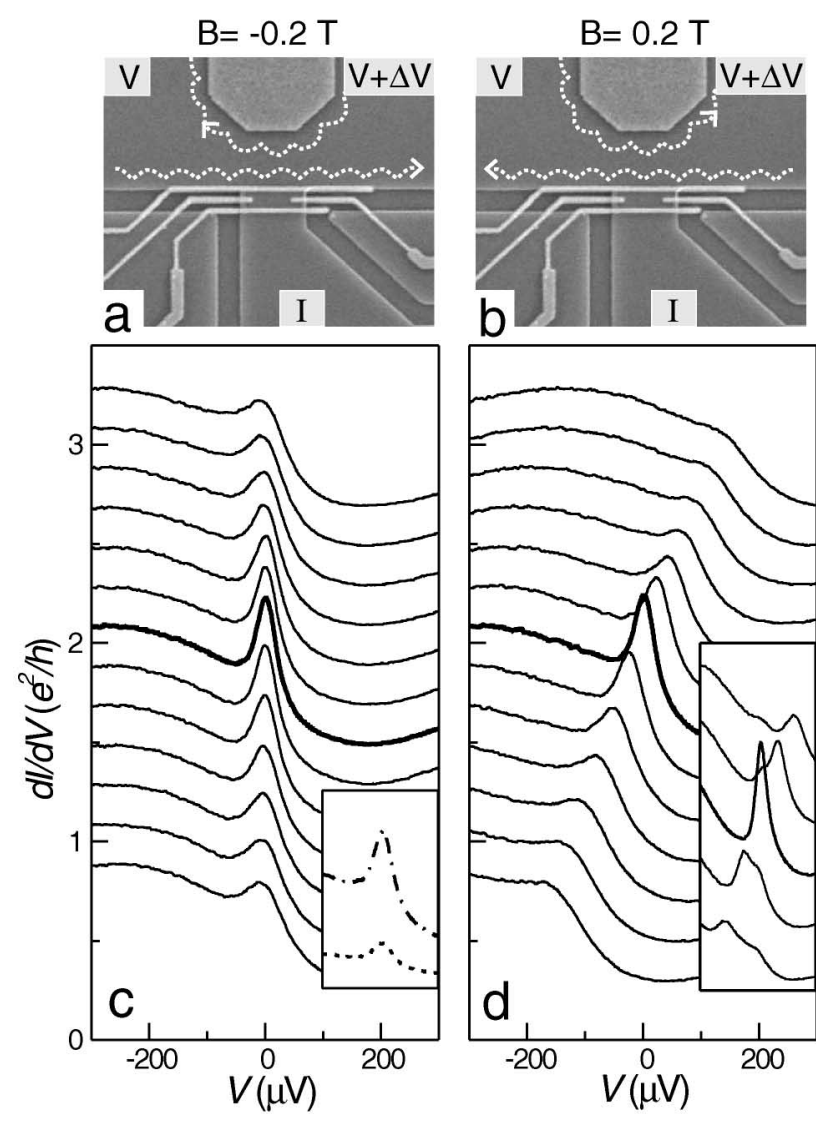

FIG. 4. (a) White dotted lines depict electron motion through the wire in the presence of a perpendicular magnetic field $B=$ $-0.2 \mathrm{~T}$. The Lorentz force pushes right movers down, closer to the dot, and left movers up, away from it. An opposite scenario occurs for $B=0.2 \mathrm{~T}$ as shown in (b). (c),(d) $d I / d V$ vs $V$, for $\Delta V$ between $-300 \mu \mathrm{V}$ (top trace) and $300 \mu \mathrm{V}$ (bottom trace) in steps of $50 \mu \mathrm{V}$. Curves are offset vertically by $0.2 e^{2} / h$ and horizontally by $0.20 \Delta V$. The inset to (c) shows that most of the current through the dot comes from the strongly coupled lead (i.e., source 1 for $B=-0.2 \mathrm{~T}$ ). The dot-dashed (dotted) trace is $d I / d V$ vs $V$, measured by the lock-in technique with the ac voltage excitation, $\tilde{\boldsymbol{v}}$, applied only to the left (right) reservoir. This allows us to directly measure the contribution from the left (right) reservoir. The horizontal axis runs from -100 to $100 \mu \mathrm{V}$, the vertical axis from 0 to $e^{2} / h$. The inset to (d) shows $d I / d V$ vs $V$ for $B=0.2 \mathrm{~T}, \Delta V$ from -150 (top) to $150 \mu \mathrm{V}$ (bottom) in steps of $50 \mu \mathrm{V}$, and a more negative voltage $V_{c h}=$ $-1.5 \mathrm{~V}$ on the top gate [see Fig. 1(c)]. Horizontal scale: from -150 to $150 \mu \mathrm{V}$. Vertical scale: from 0 to $2 e^{2} / h$. Traces are vertically offset by $0.3 e^{2} / h$.

the Kondo peaks as obtained from Figs. 3(a), 4(c), and 4(d). Split peaks from Fig. 3(a) (solid circles) match well with the single peaks from Figs. 4(c) and 4(d) (open squares and diamonds). This corroborates the interpretation given earlier that split peaks occur when $\mu_{D}$ lines up with $\mu_{S 1}$ and $\mu_{S 2}$.

In Fig. 3(c) we compare the $\Delta V$ dependence of the peak height at zero and finite $B$. The suppression of the Kondo resonance becomes considerably less severe at finite $B$.
This increased robustness stems from the displacement between left and right movers which results in a lower rate for inelastic scattering events. Negative magnetic fields appear to be more effective in this respect [15].

In conclusion, we have shown that a voltage difference between two Fermi seas coupled to a quantum dot causes the Kondo resonance to split, in agreement with theory. Split peaks are strongly suppressed for voltage differences much larger than $k_{B} T_{K} / e$. A perpendicular magnetic field has proved a useful tool to control the relative coupling of the leads. This provides a way to obtain a three-terminal quantum dot in which one of the leads is weakly coupled and can be used as a probe for the local DOS [11,12].

We thank Yu. V. Nazarov, J. Kroha, Y. Meir, L. I. Glazman, J. E. Mooij, T. Hayashi, B. van der Enden, and R. Schouten for discussions and help. This work was supported by the Specially Promoted Research, Grant-in-Aid for Scientific Research, from the Ministry of Education, Culture, Sports, Science and Technology in Japan, the Dutch Organisation for Fundamental Research on Matter (FOM), the DARPA-QUIST program (DAAD19-01-1-0659), and the EU via a TMR network (ERBFMRX CT98-0180).

[1] L. P. Kouwenhoven, C. M. Marcus, P. L. McEuen, S. Tarucha, R. M. Westervelt, and N. S. Wingreen, in Mesoscopic Electron Transport, edited by L. L. Sohn, L. P. Kouwenhoven, and G. Schön, NATO Advanced Study Institutes, Ser. E, Vol. 345 (Kluwer, Dordrecht, Boston, 1997), pp. 105-214.

[2] L. I. Glazman and M. E. Raikh, JETP Lett. 47, 452 (1988); T. K. Ng and P. A. Lee, Phys. Rev. Lett. 61, 1768 (1988).

[3] D. Goldhaber-Gordon et al., Nature (London) 391, 156 (1998); S. M. Cronenwett et al., Science 281, 540 (1998); J. Schmid et al., Physica (Amsterdam) 256B-258B, 182 (1998); F. Simmel et al., Phys. Rev. Lett. 83, 804 (1999).

[4] L. P. Kouwenhoven and L. I. Glazman, Phys. World 14, 33 (2001).

[5] Y. Meir, N. S. Wingreen, and P. A. Lee, Phys. Rev. Lett. 70, 2601 (1993).

[6] A. Kaminski, Yu. V. Nazarov, and L. I. Glazman, Phys. Rev. Lett. 83, 384 (1999); Phys. Rev. B 62, 8154 (2000).

[7] A. Rosch, J. Kroha, and P. Wölffle, Phys. Rev. Lett. 87, 156802 (2001).

[8] P. Coleman, C. Hooley, and O. Parcollet, Phys. Rev. Lett. 86, 4088 (2001).

[9] X.-G. Wen, cond-mat/9812431.

[10] Y.-W. Lee and Y.-L. Lee, Phys. Rev. B 65, 155324 (2002).

[11] Q.-F. Sun and H. Guo, Phys. Rev. B 64, 153306 (2001).

[12] E. Lebanon and A. Schiller, Phys. Rev. B 65, 035308 (2002).

[13] H. Pothier et al., Phys. Rev. Lett. 79, 3490 (1997).

[14] R. de Picciotto et al., Nature (London) 411, 51 (2001).

[15] This discrepancy may be attributed to the lack of leftright symmetry in the device. 